\title{
Electron temperature gradient driven instabilities in helical reversed-field pinch plasmas
}

\author{
I. Predebon, ${ }^{1}$ P. Xanthopoulos, ${ }^{2}$ and M. Gobbin ${ }^{1}$ \\ ${ }^{1}$ Consorzio RFX, Corso Stati Uniti 4, 35127 Padova, Italy \\ ${ }^{2}$ Max-Planck-Institut für Plasmaphysik, Wendelsteinstraße 1, 17491 Greifswald, Germany
}

(Dated: February 20, 2019)

\begin{abstract}
We describe the occurrence of electron temperature gradient driven micro-instabilities in the helical states of RFX-mod reversed-field pinch plasmas. These plasmas are usually characterized by the presence of large, radially localized electron temperature gradients. Using realistic geometry and profiles, micro-tearing modes and electron-temperature-gradient modes turn out to regularly coexist in the region of the temperature barrier. In the paper, we discuss the main features of such instabilities, especially focusing on the distinctive aspects of the helical geometry with respect to the toroidal symmetry.
\end{abstract}

\section{INTRODUCTION}

In the last years, there has been a remarkable effort to characterize the reversed-field pinch (RFP) configuration in terms of micro-instabilities and related turbulence processes. The driving motivation is the development of experimental scenarios with reduced large-scale magnetic chaos, with ensuing better confinement properties of the plasma. In the two major RFP experiments - MST and RFX-mod - this has been obtained either suppressing the overall spectrum of resonant tearing modes (by means of pulsed-poloidal-current-drive techniques, in the MST device [1]), or making the plasma evolve towards a helical state (by increasing the plasma current, letting the innermost resonant tearing mode grow until saturation, and controlling the secondary modes, in the RFX-mod device [2]).

In the previous studies, ion-temperature-gradient (ITG) mode turbulence has been investigated initially, finding a rather high stability threshold and strong zonal flows, also in the presence of impurities [3-8]. Afterwards, the direct observation of fluctuations in the mentioned experimental scenarios has been characterized in terms of microturbulence itself: trapped electron mode (TEM) for the density fluctuations in MST, driven by the high density gradients close to the edge region [9], and microtearing mode (MTM) for the high-wavenumber magnetic fluctuations in RFX-mod, driven by the electron temperature gradients in the region surrounding the helical core $[10,11]$. Concerning the latter case, we remark that the modelling was based on an axisymmetric approximation of the plasma, with the potentially critical consequences that such an approach could have on the results. In fact, the inclusion of a proper geometric treatment for the RFX-mod helical plasmas has already revealed its importance for the estimate of the ITG mode stability, zonal flows, and turbulent transport levels [12].

In this paper, indeed, we aim to address the role of micro-instabilities in the region of the electron temperature barriers in helical RFX-mod configurations, dealing with a full geometric description of the plasma and with realistic plasma profiles. For this, we make use of mag-

\begin{tabular}{|c|c|c|c|c|c|}
\hline \multicolumn{6}{|c|}{$A \quad \# 28624, t=85 \mathrm{~ms}$} \\
\hline$s$ & 0.40 & 0.45 & 0.50 & 0.55 & 0.60 \\
\hline$q$ & 0.125 & 0.123 & 0.121 & 0.117 & 0.112 \\
\hline$\hat{s}$ & -0.12 & -0.27 & -0.51 & -0.83 & -1.22 \\
\hline $1 / L_{T_{e}}$ & 2.25 & 3.49 & 4.03 & 3.50 & 2.51 \\
\hline $1 / L_{n_{e}}$ & 0.00 & 0.00 & 0.00 & 0.01 & 0.02 \\
\hline$\beta_{e}[\%]$ & 0.82 & 0.78 & 0.72 & 0.68 & 0.66 \\
\hline \multicolumn{6}{|c|}{$B \quad \# 30843, t=74 \mathrm{~ms}$} \\
\hline$s$ & 0.45 & 0.50 & 0.55 & 0.60 & 0.65 \\
\hline$q$ & 0.124 & 0.123 & 0.121 & 0.116 & 0.110 \\
\hline$\hat{s}$ & 0.01 & -0.26 & -0.66 & -1.13 & -1.70 \\
\hline $1 / L_{T_{e}}$ & 1.70 & 4.94 & 8.03 & 3.28 & 2.56 \\
\hline $1 / L_{n_{e}}$ & 0.00 & 0.01 & 0.01 & 0.02 & 0.04 \\
\hline$\beta_{e}[\%]$ & 0.74 & 0.70 & 0.59 & 0.52 & 0.52 \\
\hline
\end{tabular}

TABLE I. Main parameters for two RFX-mod experimental cases at different radii: $A$ (shot \#28624) and $B$ (shot \#30843).

netic equilibria reconstructed with the VMEC code [13], assuming magnetic surface integrity everywhere. The Eulerian gyrokinetic code GENE [14] is then used for local electromagnetic linear analyses, linked to the $3 \mathrm{D}$ equilibria by means of GIST [15], an interface code able to provide the necessary geometric information for GENE to work, mainly used for turbulence studies in the stellarator.

MTMs and electron-temperature-gradient (ETG) modes turn out to be the most unstable modes in the experimental scenarios chosen for our analysis. In this work, we will characterize their typical wavenumbers, amplitude and eigenfunction structures, as well as the dependence on some basic macroscopic parameters like the plasma $\beta$. Similarly to [12], to highlight the role of the geometry, a comparison with an axisymmetric equilibrium will be done. The entire work is focused on linear results, but a discussion on the possible consequences on electron heat transport will be included in the conclusions of the paper. 

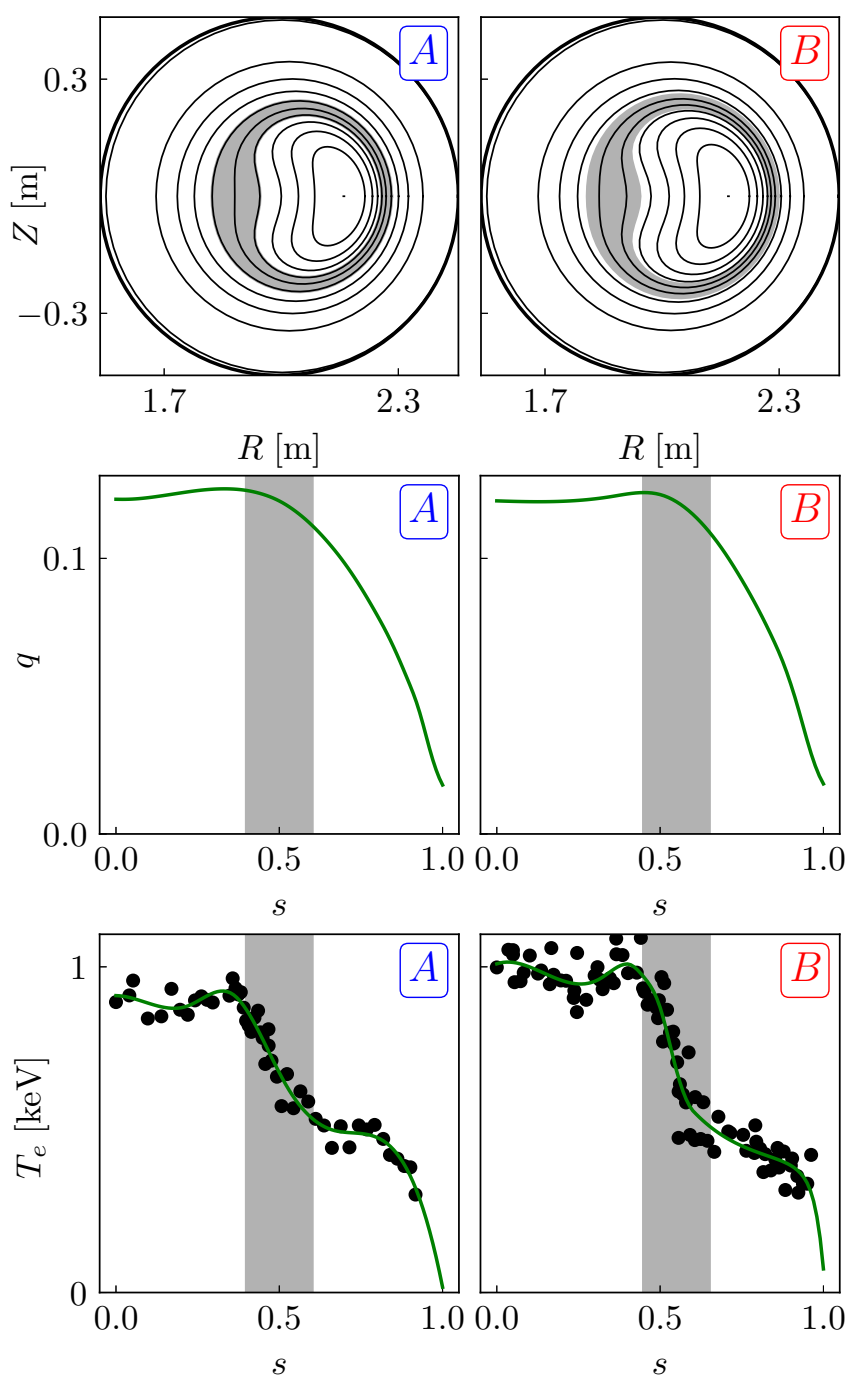

FIG. 1. RFX-mod shots \#28624, $t=85 \mathrm{~ms}$ (case $A$, left column), and \#30843, $t=74 \mathrm{~ms}$ (case $B$, right column): $\phi=0$ section of the equilibrium magnetic surfaces (1st row), $q$ profiles (2nd row) and $T_{e}$ profiles (3rd row) as a function of the normalized toroidal flux $s$. Shaded areas represent the radial region subject to gyrokinetic analysis.

\begin{tabular}{cccccc}
\hline \hline \multicolumn{5}{c}{$A_{a}(A \rightarrow$ axisymmetric $)$} \\
$s$ & 0.40 & 0.45 & 0.50 & 0.55 & 0.60 \\
\hline$q$ & 0.130 & 0.126 & 0.121 & 0.116 & 0.110 \\
$\hat{s}$ & -0.51 & -0.63 & -0.79 & -0.99 & -1.26 \\
\hline
\end{tabular}

TABLE II. Geometric parameters for the axisymmetric configuration $A_{a}$.

\section{HELICAL CONFIGURATIONS}

The helical equilibria are reconstructed with the spectral code VMEC [13]. Similarly to the approach carried out in [12], the $q$ profile is slightly modified in the plasma edge keeping it positive. This adjustment of $q$ allows us to

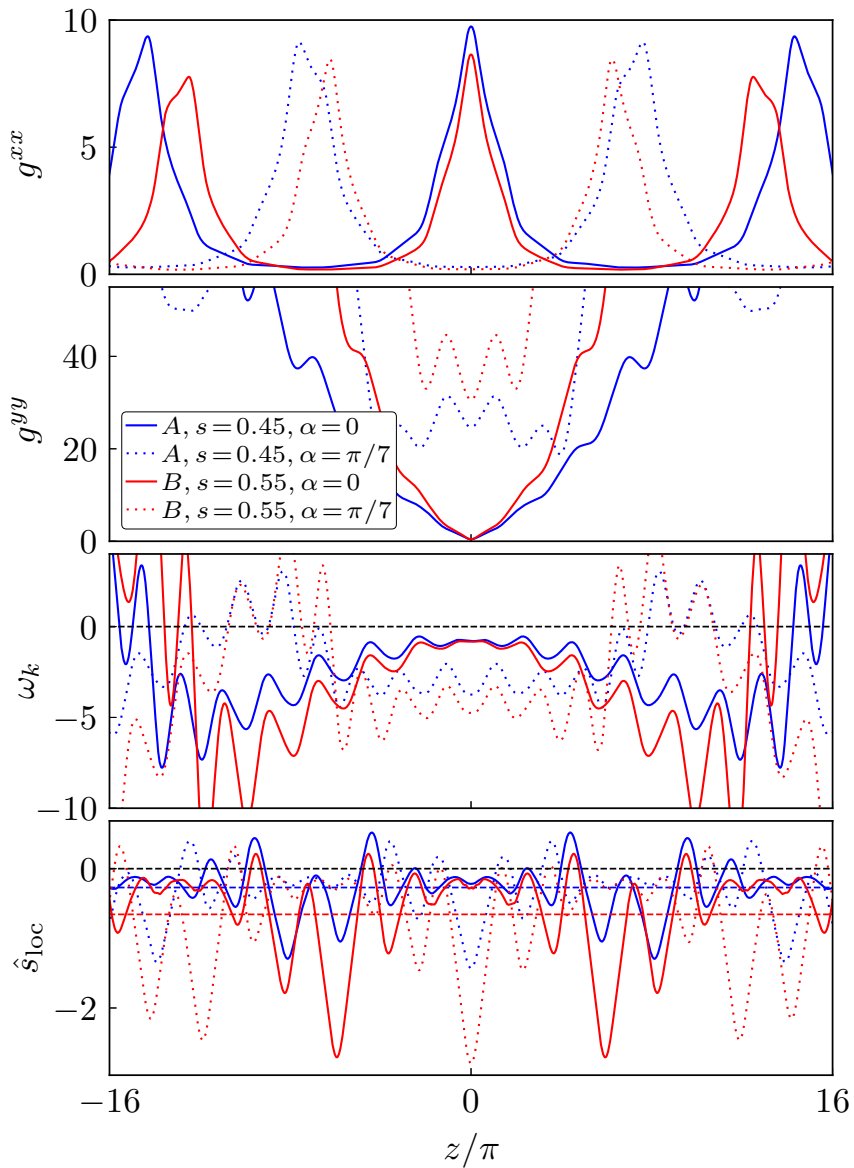

FIG. 2. Geometric coefficients vs the longitudinal coordinate $z$ for cases $\mathrm{A}$ and $\mathrm{B}$ (blue and red, respectively), for the stellarator-symmetric flux tubes $\alpha=0$ (solid lines) and $\alpha=\pi / 7$ (dotted lines). The dashed lines in the lower frame represent the global magnetic shear $\hat{s}=\left\langle\hat{s}_{\text {loc }}\right\rangle_{z}$.

use the normalized toroidal flux $s=\psi_{t} / \psi_{t \text {,edge }}$ as a radial label (which would otherwise decrease with the poloidal flux $\psi_{p}$ in the region with $q<0$ ), which is the coordinate normally used by the numerical codes interfaced to VMEC, including GIST. On the other hand, since the RFX-mod helical states are characterized by a very shallow reversal $(q(a) \gtrsim-0.01)$, this modification impacts just the last 2-3 centimeters at the edge, without altering at all the results in the core region.

In the paper, we will deal with two RFX-mod experimental cases, called $A$ and $B$, with the main features summarized in Fig. 1. The two cases are both characterized by a helical configuration with seven-fold symmetry, $(m, n)=(1,7)$, with a rather similar $q$ but different $T_{e}$ profile (the gradient is much larger in the case $B$ ). The shaded areas represent the radial regions under investigation, which are indeed characterized by the presence of large temperature barriers. The $T_{e}$ profiles are built from a mapping of the Thomson scattering data on the VMEC equilbria. The resulting $T_{e}(s)$ profiles are sufficiently well resolved at mid radius. On the other hand, 

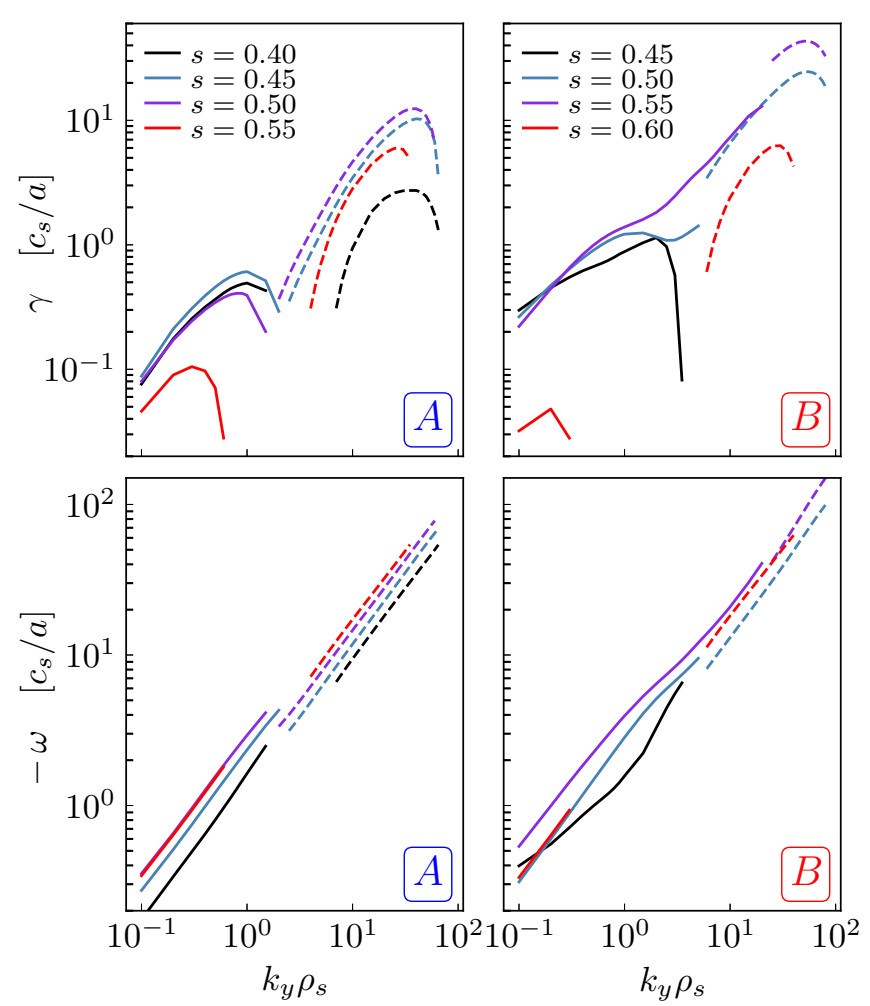

FIG. 3. Linear GENE calculations of the growth rate $\gamma$ and real frequency $\omega$ for cases A (1st column) and B (2nd column) at different radii, $\alpha=0$. Solid lines correspond to modes with tearing parity (MTM), dashed line to modes with ballooning parity (ETG).

the ion temperature is only partially known; based on spectroscopic and neutral-particle-analyzer data [16], for its profile we assume $T_{i} / T_{e} \sim 0.7$ in the core and $T_{e} \sim T_{i}$ in the edge, with a resulting lower ion temperature gradient (approximately halved, in the region of the electron temperature barrier); concerning the following gyrokinetic results, we remark (and anticipate) that their robustness has been checked against different plausible $T_{i}$ profiles, showing that the unstable modes (mainly MTM and ETG) are scarcely coupled to ITG modes, which are sub-dominant. Finally, the electron density profile $n_{e}(s)$ is almost flat, as usual in most of the RFX-mod experimental scenarios, including the helical states [16]. Some important parameters for the gyrokinetic analysis are reported in Table I. Here, the (global) magnetic shear is defined as $\hat{s}=2 s q^{\prime}(s) / q$, the electron temperature and density gradient as $1 / L_{T_{e}}=-T_{e}^{-1} d T_{e} / d \sqrt{s}$ and $1 / L_{n_{e}}=-n_{e}^{-1} d n_{e} / d \sqrt{s}$, respectively, and the electron plasma beta as $\beta_{e}=8 \pi n_{e} T_{e} /\langle B\rangle^{2}$, where $\langle\cdot\rangle$ is the average on the surface $s=$ const.

For the reconstruction of the flux tube domain, we rely on the code GIST [15]. GIST provides a full geometric description of the metric tensor, curvature, Jacobian and parallel gradients needed for the gyrokinetic code GENE [14] to work. Starting from the VMEC co-

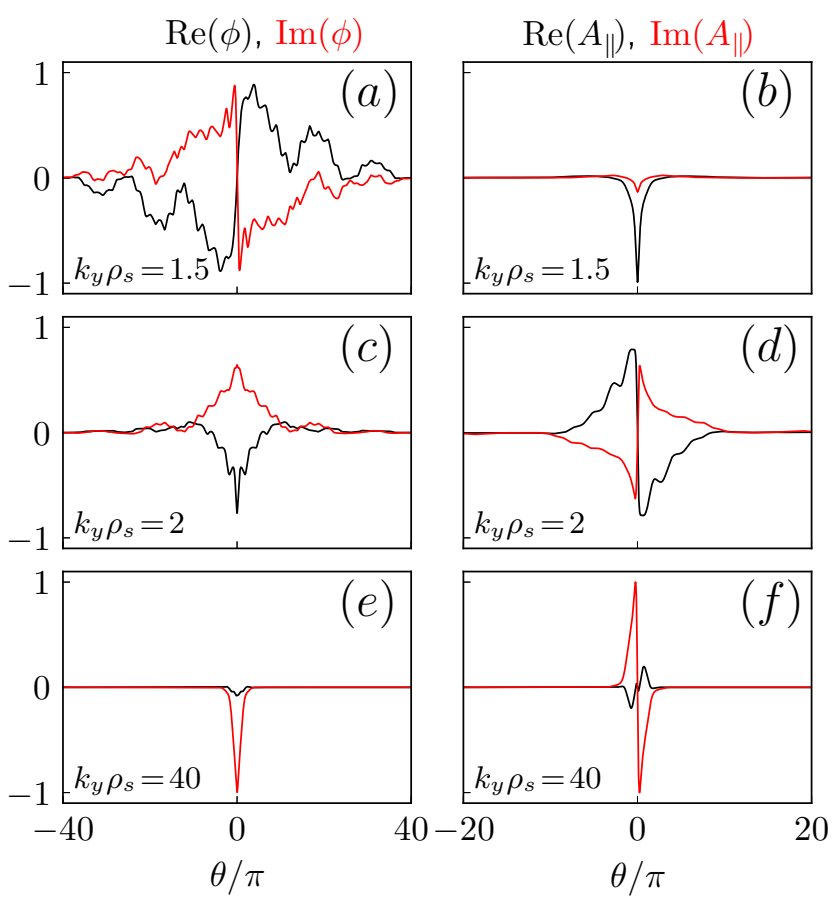

FIG. 4. $\phi$ and $A_{\|}$eigenfunctions for case A at $s=0.5$, for different wavenumbers $k_{y} \rho_{s}$.

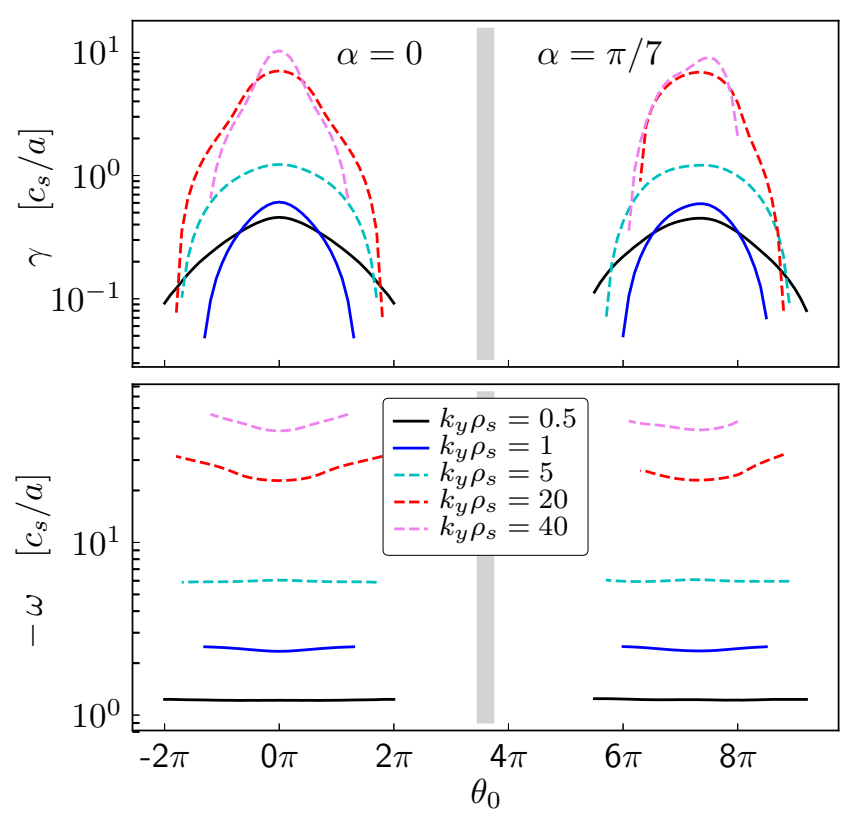

FIG. 5. Growth rate and real frequency as a function of the ballooning angle $\theta_{0}$ for case $\mathrm{A}, s=0.45$, at different binormal wavenumbers $k_{y} \rho_{s}$, for the two stellarator-symmetric tubes $\alpha=0$ (left) and $\alpha=\pi / 7$ (right). Solid lines correspond to modes with tearing parity (MTM), dashed line to modes with ballooning parity (ETG).

ordinates $(s, \vartheta, \phi)$, with $\vartheta$ and $\phi$ the VMEC poloidal angle and the cylindrical toroidal angle, the straight-fieldline poloidal angle is built from the VMEC stream func- 


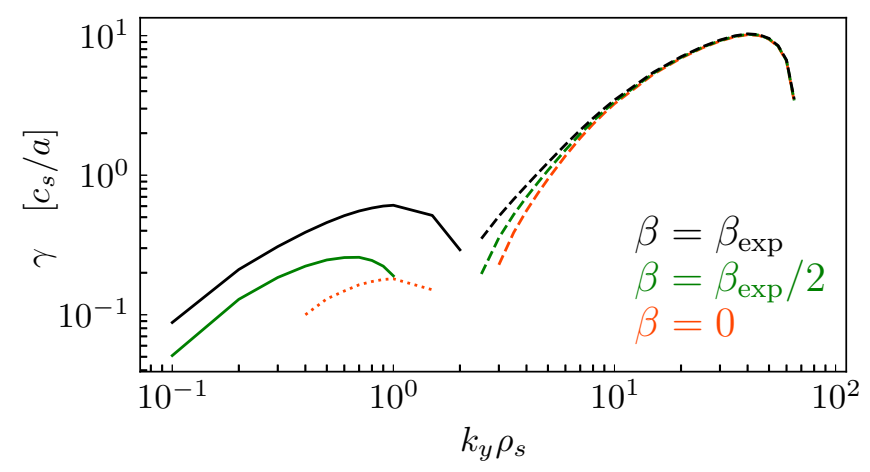

FIG. 6. Dependence of the growth rate on $k_{y} \rho_{s}$ for different values of $\beta$ (nominal experimental value, half of its value, and zero), for case $A, s=0.45, \alpha=0$ flux tube. Solid lines correspond to modes with tearing parity (MTM), dashed line to modes with ballooning parity (ETG). The dotted line represents modes with ballooning parity and positive real frequency (ITG), appearing for $\beta=0$ at low $k_{y} \rho_{s}$.

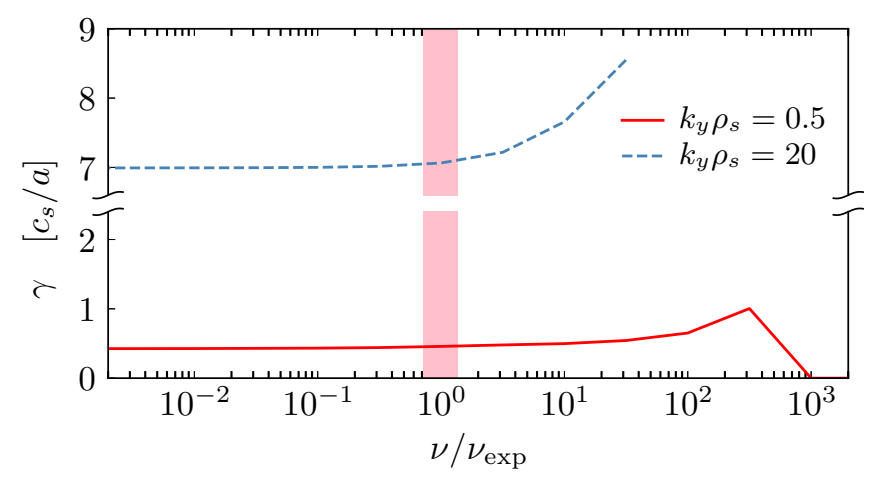

FIG. 7. Growth rate as a function of the collisionality normalized to the experimental value for case $A, s=0.45, \alpha=0$ flux tube. As usual, solid lines correspond to modes with tearing parity (MTM), dashed line to modes with ballooning parity (ETG).

tion $\lambda, \theta=\vartheta+\lambda(s, \vartheta, \phi)$. The GIST coordinates are triplets $(x, y, z)$, defined as $x=s^{1 / 2}$ (radial coordinate), $y=\left.\left.\left(s^{1 / 2} / q\right)(q \theta-\phi)\right|_{s=s_{0}} \equiv\left(s^{1 / 2} / q\right)\right|_{s=s_{0}} \alpha$ (binormal coordinate), $z=\theta$ (parallel coordinate).

As extensively discussed in [12], two stellaratorsymmetric flux-tubes can be reconstructed in our helical state with seven-fold symmetry, $(m, n)=(1,7)$, one with $\alpha=0$ and the other with $\alpha=\pi / 7$, centered in the outboard mid-plane at $\phi=0$ section and in the outboard mid-plane at $\phi=-\pi / 7$, respectively. In Fig. 2, some important geometric quantities are shown as a function of $z$ for the $\alpha=0$ and $\alpha=\pi / 7$ tubes, for $A$ and $B$; they are the $g^{x x}$ and $g^{y y}$ components of the metric tensor, the curvature $\omega_{k}=\left(\hat{B} \sqrt{g^{x x}} \kappa_{\text {norm }}+g^{x y} \kappa_{\text {geo }}\right) / g^{x x}$ (with $\kappa_{\text {norm }}$ and $\kappa_{\text {geo }}$ the normal and geodesic components of the field line curvature, respectively), and the local magnetic shear $\hat{s}_{\text {loc }}=d\left(g^{x y} / g^{x x}\right) / d z$, with the dashed lines representing the values of the (global) magnetic shear, given by $\hat{s}=\left\langle\hat{s}_{\text {loc }}\right\rangle_{z}=2 s q^{\prime}(s) / q$. The stellarator sym-
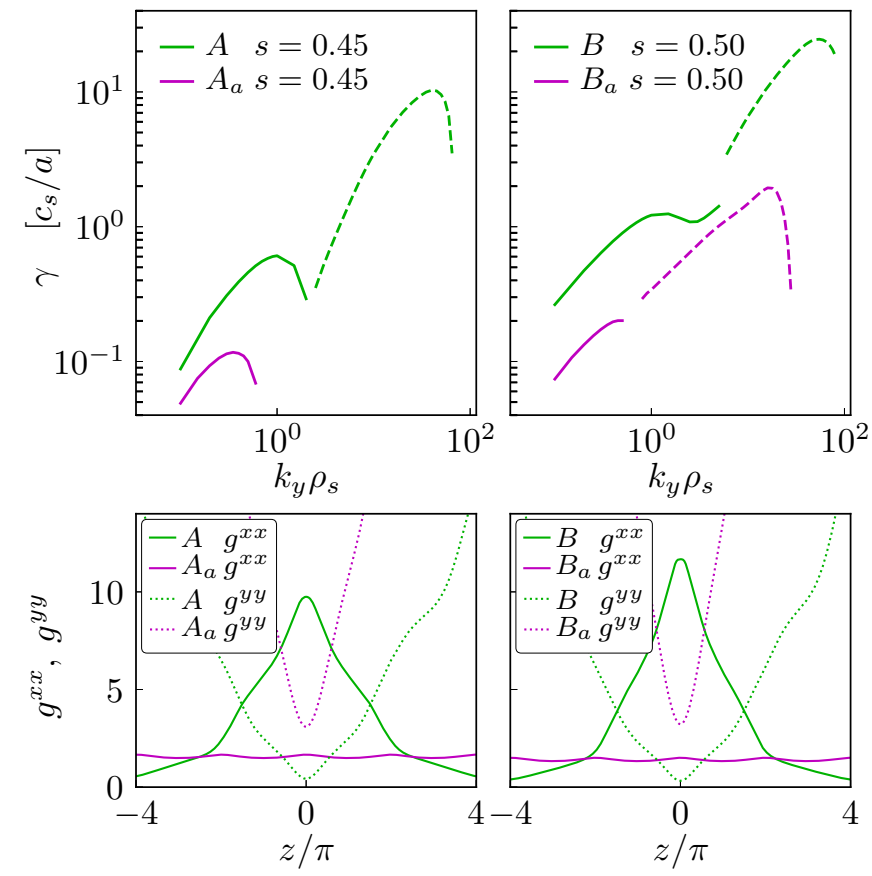

FIG. 8. Comparison of helical $(\alpha=0)$ and axisymmetric growth rates (upper frames) and corresponding geometric coefficients (lower frames), case A (left) and B (right). In the upper frames, solid lines correspond to modes with tearing parity (MTM), dashed line to modes with ballooning parity (ETG).

metry [17] of the flux tubes is indeed characterized by the even symmetry of the above quantities with respect to the longitudinal coordinate, and odd for other coefficients, e.g., $g^{x y}$. In Sec. IIID we will discuss also the comparison with an axisymmetric configuration. In that case, the dependence on the binormal coordinate $\alpha$ is absent, i.e., every field line on an axisymmetric surface has the same geometric features.

\section{GYROKINETIC INVESTIGATIONS}

\section{A. Linear analysis with nominal plasma parameters}

Based on the previously defined flux tube domain, the code GENE can now be used to solve the gyrokinetic equation in realistic conditions. Our linear simulations include two fully kinetic species, without impurities. In order to resolve elongated eigenfunctions and long tails in the electrostatic potential, a longitudinal domain $z \in[-48 \pi,+48 \pi]$ to $z \in[-64 \pi,+64 \pi]$ has been assumed, depending on the radial position and on the related global magnetic shear, with a discretization of 64 grid points per poloidal turn. In the parallel velocity and magnetic moment space, we set $\left(v_{\|}, \mu\right) \in\left[-3 v_{\text {th }}, 3 v_{\text {th }}\right] \times[0,9 T / B]$, with $64 \times 16$ grid points. Parallel vector potential fluctuations are included, as well as the electrostatic potential 

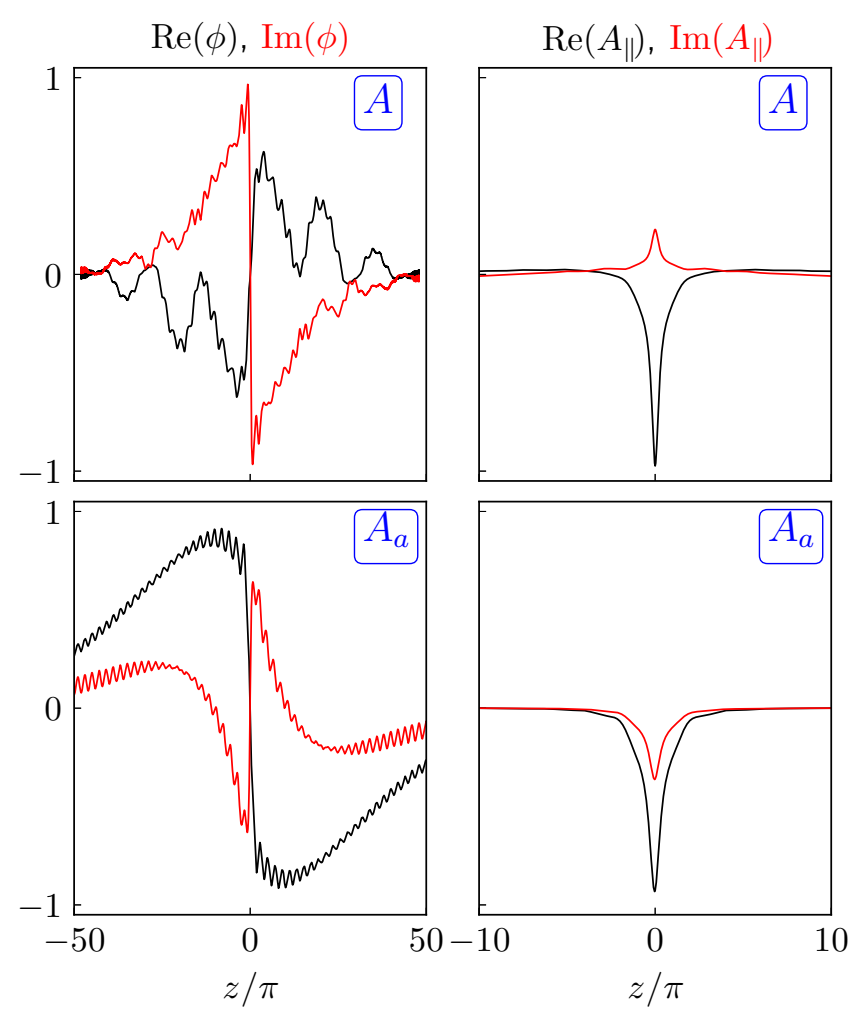

FIG. 9. (1st row) $\phi$ and $A_{\|}$MTM eigenfunctions for case A, $s=0.45, k_{y} \rho_{s}=1.5$, and (2nd row) comparison with the related axisymmetric geometric, $s=0.5, k_{y} \rho_{s}=0.5$.

ones. For our simulations we use a Landau-Boltzmann collision operator, and the collision frequency is calculated assuming $Z_{\text {eff }}=1.6$, where $Z_{\text {eff }}=\sum_{j} n_{j} Z_{j}^{2} / n_{e}$ is the effective ion charge considering all the present ion species $j$ with charge state $Z_{j}$ and respective density $n_{j}$. Convergence tests have been done to establish the correct range of hyper-diffusion, in particular in the longitudinal coordinate $z$ in order to damp unphysical oscillations at high $|z|$ without affecting much the growth rate and real frequency of the mode. We investigate the occurrence of both tearing parity modes, with $\phi(-x, y,-z)=-\phi(x, y, z), A_{\|}(-x, y,-z)=A_{\|}(x, y, z)$, and interchange parity modes, with opposite parity.

Out of the shaded region of Fig. 1, an overall stability of the modes is found: the temperature gradients are moderate, and the density profile is practically flat, with $1 / L_{n_{e}}<0.1$ everywhere for $s<0.8$, for both case $A$ and $B$. With such profiles, density gradient driven TEMs are stable. As reported in [9], these modes become important for large $1 / L_{n_{e}}$, but in RFX-mod the density gradients are steep only in the very edge of the plasma, which is a region out of the scope of this work.

Let us consider the shaded area of Fig. 1. Here, for the $\alpha=0$ flux tube, a large set of modes is destabilized by the temperature gradient, as shown in Fig. 3. Let us investigate the case with $k_{x}=0$ first. We have two branches, both characterized by modes propagat- ing in the electron diamagnetic direction, $\omega<0$. At low wavenumber, $k_{y} \rho_{i}<1-2$, the modes are identified as MTMs. They have tearing parity, as shown in Fig. 4, with elongated structure of the electrostatic potential, and much more localized in $A_{\|}$. Furthermore, the growth rate increases with $1 / L_{T_{e}}$ and $\beta$ (the latter dependency will be discussed in Sec. III B). The real frequency is of the order of electron diamagnetic frequency $\omega_{* e}$. For higher wavenumbers, ETG modes are dominant, with opposite parity (even in $\phi$, odd in $A_{\|}$). In the lower wavenumber range of this branch, $k_{y} \rho_{i} \sim 2$, the structure is still very elongated, mainly due to the low value of the global magnetic shear. Around the peak wavenumber, the structure is on the contrary more localized. Comparing the two experimental cases, in $A$ the two branches are well separated, whereas in $B$, in particular at the radius with the largest $\nabla T_{e}$, the MTM and ETG branches are mixed: MTMs exist until large wavenumbers, and the parity of the modes switches without a large change in the real frequency.

Still focusing on the case $k_{x}=0$, the entire set of modes is stable for the $\alpha=\pi / 7$ flux tube. Thus the partial conclusion is that the modes are strongly unstable for the tube centered around the region with higher flux surface proximity $(\alpha=0)$ and stable where the magnetic surfaces are less densely packed $(\alpha=\pi / 7)$. However, dealing only with vanishing $k_{x}$ 's may lead to wrong conclusions. In fact, expressing the radial wavenumber as $k_{x}=-k_{y} \hat{s} \theta_{0}$, if we look at the stability of the modes as a function of the ballooning angle $\theta_{0}$, growth rates comparable to the previous ones are found for non-vanishing $\theta_{0}$ in the case $\alpha=\pi / 7$, see Fig. 5. For $\alpha=0$, a localization of positive growth rates occurs for $-2 \pi \lesssim \theta_{0} \lesssim+2 \pi$ with a peak at $\theta_{0}=0$, both for MTMs and ETG modes. On the other hand, for $\alpha=\pi / 7$, the structure turns out to be shifted to $5 \pi \lesssim \theta_{0} \lesssim 9 \pi$, peaking between $7 \pi$ and $8 \pi$. A similar localization is found for the neighboring surfaces, depending on the value of $q$.

Without repeating the whole study in [12], mode structure and stability are strictly linked to the behaviour of $k_{\perp}=\left(g^{y y}-2 \hat{s} \theta_{0} g^{x y}+\hat{s}^{2} \theta_{0}^{2} g^{x x}\right)^{1 / 2} k_{y}$ along the fluxtube, in particular to its minima and the possible jumps along the flux-tube, which are able to introduce hard boundaries for the eigenfunctions via finite Larmor radius (FLR) suppression. To this aim, we recall that FLR suppression of the modes occurs through the Bessel terms $J_{0}\left(k_{\perp} v_{\perp} / \Omega\right)$ in the linear gyrokinetic equation.

For $\alpha=0$, the minimum of $k_{\perp}$ occurs at $\theta_{0}=0$, where $\min \left(k_{\perp} / k_{y}\right)=\left(\left.g^{y y}\right|_{z=0}\right)^{1 / 2}(\simeq 0.65$ for the case in Fig. 5, left side). For $\alpha=\pi / 7$, the minimum occurs for a finite $\theta_{0}$, off-center in $z$ (the minimum is $\simeq 0.71$ at $\theta_{0} \simeq 7.57 \pi$, see right side of Fig. 5). As is evident in the figure, the growth rates and the real frequencies corresponding to the peak values are comparable for the two flux-tubes, being slightly larger $(2-10 \%)$ in the $\alpha=0$ case, ultimately due to the toroidicity of the system. We will compare these results with the corresponding ones in axisymmetry in Sec. III D. 
We remark here that the localization in wavenumber naturally brings to a localization in the real space. The combination of the $g^{i j}$ components in the expression of $k_{\perp}$ allows to identify the local maxima of $g^{x x}$ as a key responsible for the local destabilization of the modes. Even in the case $\theta_{0}=0$ (which prevents $g^{x x}$ from entering directly the linear gyrokinetic equation), the minimum of $g^{y y}$ is strictly related to a maximum of $g^{x x}$, as $g^{y y}=\left(B^{2}+\left(g^{x y}\right)^{1 / 2}\right) / g^{x x}$ in any field-aligned Clebsch system. In the real space, the maxima of $g^{x x}=|\nabla x|^{2}$ correspond to the regions with highest magnetic surface proximity. This proximity is higher along the helical ridge of the magnetic surface, and lower on the opposite side, where the surfaces are rarefied. Heuristically, in the real space, densely packed surfaces cause the gradients (measured in $\mathrm{m}^{-1}$ ) to increase, hence the instabilities to grow. Finally, we point out that, in a helical RFP, the tensor component $g^{x x}(z)$ is mainly affected by the helical deformation and much less by toroidicity, which explains the minor difference in growth rate and real frequency between the two flux tubes in Fig. 5, as mentioned above.

\section{B. Dependence on $\beta$}

Microtearing modes are electromagnetic in nature, therefore they are expected to be strongly dependent on $\beta$. This dependence has been investigated in several papers, with increasing $\beta$ found, in general, to be destabilizing until a peak value, and stabilizing afterwards. This has been discussed, e.g., in [18] for a slab geometry, and [19] for a spherical tokamak. Here, in Fig. 6, we limit the analysis to experiment-like values, first keeping the nominal $\beta$, then halving its value, and finally setting $\beta=0$. If $\beta=\beta_{\exp } / 2$, the growth rate of the MTM branch is approximately halved with respect to the case with experimental $\beta$ (solid lines). In the electrostatic limit $\beta=0$, as expected, MTMs are suppressed; here, an ITG branch emerges, driven by the non-vanishing ion temperature gradient (dotted line).

ETG modes (dashed lines) are destabilized by a finite $\beta$ especially in the low wavenumber range, $2 \lesssim k_{y} \rho_{i} \lesssim 10$, while they are scarcely destabilized at peak wavenumbers and beyond: $\max (\gamma)_{\beta=\beta_{\text {exp }}}$ and $\max (\gamma)_{\beta=\beta_{\exp } / 2}$ differ by $0.73 \%$, and the difference becomes $1.45 \%$ when comparing the maximum $\gamma$ at $\beta_{\text {exp }}$ and $\beta=0$. We remark that a similar slight destabilization of ETG modes has been found in previous gyrokinetic simulations in the tokamak core [20] (isolating the effect of magnetic fluctuations from that on the equilibrium), while older analytic works have shown a stronger finite- $\beta$ destabilization of the toroidal ETG branch [21], in contrast to the stabilizing electromagnetic effect in slab geometry.

\section{Dependence on collisionality}

Now we artificially (and inconsistently) change the collisionality while keeping the other parameters fixed, to further characterize the instabilities. The result is shown in Fig. 7 for two wavenumbers, one corresponding to the MTM branch, the other to the ETG one. MTMs present the usual trend versus $\nu_{e i}$, with a peak at large collisionality and stabilization afterwards [18]. In the collisionless limit, stabilization is expected for the slab MTM. Conversely, $\gamma>0$ is found here, in agreement with other works dealing with realistic geometries, e.g., [7, 22-25].

ETG modes are destabilized by increasing collisionalities, again in general agreement with [20]. We remark that in the region $\nu_{e i} \sim \nu_{e i, \exp }$, the dependence on the collisionality itself is weak, and that a more important destabilization is obtained for much more collisional plasmas.

\section{Role of the geometry}

In the paper [12], we compared helical and axisymmetric RFP equilibria in detail, focusing on the role of the geometric coefficients for the evaluation of ITG instabilities and turbulence. Here, we partly repeat that study with the same methodology, but focusing only on instabilities driven by the electron temperature gradient.

Starting from the helical configurations $A$ and $B$, we build two axisymmetric VMEC equilibria using the $q(s)$ profiles obtained from the toroidal equilibrium model described in [26]. The axisymmetric reconstructions provide monotonically decreasing $q$ profiles with an associated negative magnetic shear everywhere across the radius. We call these cases $A_{a}$ and $B_{a}$, respectively, where the subscript " $a$ " stands for axisymmetric. For such new configurations, we impose the same plasma profiles as in the helical configurations, i.e., the same functions $n_{e}(s)$ and $T_{e / i}(s)$ (and related macroscopic quantities, like the gradients $1 / L_{T_{e} i}(s)$ and $1 / L_{n_{e}}(s)$, and the plasma $\beta(s)$ ), where $s$ is now the normalized axisymmetric toroidal flux. The idea here is to disentangle pure geometric effects from profile effects, allowing to identify the different geometric mechanisms playing a role in the stability of the modes, and eventually in controlling the turbulence levels. The distinctive global parameters with respect to the helical cases, i.e., $q$ and the magnetic shear $\hat{s}$, are summarized in Table II for case $A_{a}$.

The comparison between helical and axisymmetric GENE results is shown in Fig. 8, where also the comparison of the $g^{x x}$ and $g^{y y}$ metric tensor components is illustrated. Here, for simplicity, we focus on a single radial position. As is clear, the whole range of modes is stabilized by moving to axisymmetry: for both cases, MTMs and ETG modes are largely damped; indeed, in case $A$ we have a complete stabilization of small scale ETG modes. Thus, as for the ITG counterpart, the effect of a change of geometry turns out to be very signif- 
icant for the evaluation of mode stability, and could be even more meaningful in a nonlinear context. There are global (flux-surface) and local (flux-tube) features associated with the two configurations, which we are going to discuss briefly together with their implications on mode stability.

The most important global (flux-surface) feature is the role of the magnetic shear $\hat{s}$. For the same surface $s$, the magnetic shear differs in the two geometries. Especially in the region of the electron temperature barrier, the helical $|\hat{s}|$ is lower - vanishing somewhere, as shown in [27] - than the axisymmetric shear. In the RFP, a large $|\hat{s}|$, i.e., a small magnetic shear length $L_{s}$, provides both MTM and ETG mode stabilization. As for the other microinstabilities, the magnetic shear in general affects the MTM stability, this dependence being largely determined by the background geometry under consideration: in a slab plasma the instability grows with the shear until a certain $\hat{s}_{\max }>0$, with a stabilization afterwards [18]; a similar trend is found in the RFP [23] and in the pedestal top of MAST [22], while for the core of NSTX the most unstable MTM is at $\hat{s} \sim 0$ [28]. Concerning the ETG instability, the effect of an increasing $\hat{s}>0$ is to stabilize the mode in the tokamak [20]; in the RFP, ETG modes are generally stabilized for increasing $|\hat{s}|$ (i.e., regardless of the sign of $\hat{s}$ ), as has been checked also with GS2 applied to the simplified equilibrium model of [5].

The local (flux-tube) features associated with the two classes of equilibria - helical and axisymmetric - comprise the dependence of the metric coefficients $g^{i j}$ and curvature $\omega_{k}$ on the longitudinal coordinate $z$. As we have seen in Sec. III A, in the helical geometry the higher growth rates are strictly linked to the larger magnetic surface proximity; the modes are more unstable than in axisymmetry, but localized in the radial wavenumber $k_{x}$. Conversely, the axisymmetric results do not depend on a specific flux-tube (i.e., on $\alpha$ ), and weakly depend on $\theta_{0}$, with $\gamma\left(\theta_{0}\right)$ and $\omega\left(\theta_{0}\right)$ oscillating between a maximum at $\theta_{0}=0$ and a minimum at $\theta_{0}= \pm \pi$, both for MTM and ETG modes. The evaluation of the ratio $k_{\perp} / k_{y}$ yields, for $\theta_{0}=0, \min \left(k_{\perp} / k_{y}\right)=\left(\left.g^{y y}\right|_{z=0}\right)^{1 / 2} \simeq 1.76$ in the axisymmetric case $A_{a}$ shown in Fig. 8 (left), to be compared with the value $\left(\left.g^{y y}\right|_{z=0}\right)^{1 / 2} \simeq 0.65$ of the corresponding helical configuration $A$ at the same radius $s$, as seen in Sec. III A. This, together with the shape of amplification factor $\left|k_{\perp}(z)\right| /\left|k_{\perp}(0)\right|$ implies a more effective FLR suppression of the modes in axisymmetry.

Summarizing this section, axisymmetric RFPs are characterized by lower growth rates and weak dependence on the radial wavenumber. In the real space, this corresponds to a weak toroidal ballooning structure centered around the outboard midplane. The distinctive footprint of the helical RFP is, on the other hand, a sharp helical ballooning structure. In order to understand the consequences on the net heat fluxes and transport coefficients, a nonlinear analysis is needed, which represents a very challenging computational problem due to the interplay between ion and electron Larmor radius scales, to be addressed in a fully $3 \mathrm{D}$ context.

\section{CONCLUSIONS}

In the region of the electron transport barrier, the helical states of RFX-mod reversed-field pinch plasmas turn out to be characterized by the simultaneous presence of MTMs and ETG modes. Based on the cases investigated in the paper, the modes have separated scales if the electron temperature gradients have moderate values, and become coupled when the gradients are large enough. Therefore, although the helical states are certainly appealing in terms of reduced MHD turbulence and overall better plasma performance, the large electron temperature gradients result in a drive for instabilities and turbulence extending from $\rho_{e}$ to $\rho_{i}$ scales, especially effective in the electron heat channel. The ion heat diffusivity is negligible for both MTM and ETG turbulence indeed, as well as the resulting particle diffusion, $\chi_{i} / \chi_{e} \ll 1$, $D / \chi_{e} \ll 1$.

The linear analysis made in this work focuses on some global/local properties of the modes, on the dependence on some crucial parameters like the plasma $\beta$ and the collisionality, and on the role played by the geometry. Summarizing, the low magnetic shear across the barrier destabilizes both MTMs and ETG modes, and the modes themselves turn out to have a helical ballooning structure along the flux tubes, being more unstable where the flux surface proximity is higher. This is somewhat expected, as a high surface proximity reflects on higher temperature gradients in the real space, which is the basic drive for the instabilities we are considering.

Compared to an axisymmetric configuration with the same density and temperature profiles (i.e., changing only the safety factor and the geometric coefficients), the helical states are more prone to MTM and ETG instabilites. However, this analysis does not allow to draw conclusions on the transport levels in the two geometries: to shed light on the question "are helical configurations better or worse than axisymmetric ones, in terms of micro-turbulence driven transport?" we should perform nonlinear simulations at least in the two stellaratorsymmetric tubes and compare the resulting coefficients to an axisymmetric case. As we saw in the ITG turbulence case [12], the geometry plays an important role also in the nonlinear processes (zonal flows, streamers, etc.), and in the overall structure of the fluctuating field along the flux tubes. A similar multiscale problem, with coexisting MTM and ETG turbulence, has been recently tackled in [29] for a tokamak plasma: there, due to the breaking of the MTM current sheet structures caused by electron-scale $E \times B$ flows, the MTM flutter electron heat transport turns out to be largely suppressed by the ETG component. In our case, the plasma parameters are different and the influence of the geometry could be important, so a conclusive answer would come from ad hoc 3D multi-scale simulations, but we are still far form 
that objective.

[1] J. S. Sarff, et al., Nucl. Fusion 55 (2015) 104006.

[2] R. Lorenzini, et al., Nat. Phys. 5, 570 (2009).

[3] S. C. Guo, Phys. Plasmas 15, 122510 (2008).

[4] F. Sattin, X. Garbet and S. C. Guo, Plasma Phys. Control. Fusion 52, 105002 (2010).

[5] I. Predebon, C. Angioni, and S. C. Guo, Phys. Plasmas 17, 012304 (2010).

[6] V. Tangri, P. W. Terry and R. E. Waltz, Phys. Plasmas 18, 052310 (2011).

[7] D. Carmody, M. J. Pueschel and P. W. Terry, Phys. Plasmas 20, 052110 (2013).

[8] I. Predebon, L. Carraro and C. Angioni, Plasma Phys. Control. Fusion 53, 125009 (2011).

[9] J. R. Duff, Z. R. Williams, D. L. Brower, B. E. Chapman, W. X. Ding, M. J. Pueschel, J. S. Sarff, and P. W. Terry, Phys. Plasmas 25, 010701 (2018).

[10] I. Predebon, F. Sattin, M. Veranda, D. Bonfiglio, and S. Cappello, Phys. Rev. Lett. 105, 195001 (2010).

[11] M. Zuin, S. Spagnolo, I. Predebon, F. Sattin, F. Auriemma, R. Cavazzana, A. Fassina, E. Martines, R. Paccagnella, M. Spolaore, and N. Vianello, Phys. Rev. Lett. 110, 055002 (2013).

[12] I. Predebon and P. Xanthopoulos, Phys. Plasmas 22, 052308 (2015).

[13] S. P. Hirshman and J. C. Whitson Phys. Fluids 26, 3553 (1983).

[14] F. Jenko, W. Dorland, M. Kotschenreuther, and B. N. Rogers, Phys. Plasmas 7, 1904 (2000).

[15] P. Xanthopoulos, W. A. Cooper, F. Jenko, Y. Turkin, A. Runov, J. Geiger, Phys. Plasmas 16, 082303 (2009).

[16] F. Auriemma, R. Lorenzini, M. Agostini, L. Carraro, G. De Masi, A. Fassina, M. Gobbin, E. Martines, P. Inno- cente, P. Scarin, W. Schneider, M. Zuin, Nucl Fusion 55, 043010 (2015).

[17] R. L. Dewar and S. R. Hudson, Physica D112, 275-280 (1998).

[18] N. T. Gladd, J. F. Drake, C. L. Chang, and C. S. Liu, Phys. Fluids 23, 1182 (1980).

[19] D. J. Applegate et al., Plasma Phys. Control. Fusion 49, 1113 (2007).

[20] F. Jenko, W. Dorland, and G. W. Hammett, Phys. Plasmas 8, 4096 (2001).

[21] J. Y. Kim and W. Horton, Phys. Fluids B 3, 3194 (1991).

[22] D. Dickinson, C. M. Roach, S. Saarelma, R. Scannell, A. Kirk and H. R Wilson, Plasma Phys. Control. Fusion 55 074006 (2013).

[23] I. Predebon and F. Sattin, Phys. Plasmas 20, 040701 (2013).

[24] J. Chowdhury, Yang Chen, Weigang Wan, Scott E. Parker, W. Guttenfelder, and J. M. Canik, Phys. Plasmas 23, 012513 (2016).

[25] A. K. Swamy, R. Ganesh, J. Chowdhury, S. Brunner, J. Vaclavik, and L. Villard, Phys. Plasmas 21, 082513 (2014).

[26] P. Zanca and D. Terranova, Plasma Phys. Control. Fusion 46, 1115 (2004).

[27] M. Gobbin, D. Bonfiglio, D. F. Escande, A. Fassina, L. Marrelli, A. Alfier, E. Martines, B. Momo, D. Terranova, Phys. Rev. Lett. 106, 025001 (2011).

[28] W. Guttenfelder, J. Candy, S. M. Kaye, W. M. Nevins, R. E. Bell, G. W. Hammett, B. P. LeBlanc, and H. Yuh, Phys. Plasmas 19, 022506 (2012).

[29] S. Maeyama, T.-H. Watanabe, and A. Ishizawa, Phys. Rev. Lett. 119, 195002 (2017). 